\title{
SPIE Proceedings: Laser-plasma generated very high energy electrons (VHEEs) in radiotherapy
}

\author{
K. Kokurewicz ${ }^{1 \mathrm{a})}$, G. H. Welsh ${ }^{1 \mathrm{~b})}$, E. Brunetti ${ }^{1 \mathrm{c})}$, S. M. Wiggins ${ }^{1 \mathrm{~d})}$, M. Boyd ${ }^{2 \mathrm{a})}$, A. Sorensen $^{2 \mathrm{~b})}$, \\ A. Chalmers ${ }^{3 a)}$, G. Schettino ${ }^{4 a)}$, A. Subiel ${ }^{4 b)}$, C. DesRosiers ${ }^{5 a)}$, and D. A. Jaroszynski ${ }^{1 e)}$ \\ ${ }^{1}$ University of Strathclyde, G4 0NG, Glasgow, United Kingdom \\ ${ }^{2}$ Strathclyde Institute of Pharmacy and Biomedical Sciences, G4 0RE, Glasgow, UK \\ ${ }^{3}$ Wolfson Wohl Cancer Research Centre, Institute of Cancer Sciences, Glasgow, G61 1QH, UK \\ ${ }^{4}$ National Physical Laboratory, Hampton Road, TW11 0LW, Teddington, Middlesex, UK \\ ${ }^{5}$ Indiana University, School of Medicine Department of Radiation Oncology, 535 Barnhill \\ Drive, RT 041, Indianapolis, US
}

\begin{abstract}
As an alternative modality to conventional radiotherapy, electrons with energies above $50 \mathrm{MeV}$ penetrate deeply into tissue, where the dose can be absorbed within a tumour volume with a relatively small penumbra. We investigate the physical properties of VHEEs and review the state-of-the-art in treatment planning and dosimetry. We discuss the advantages of using a laser wakefield accelerator (LWFA) and present the characteristic features of the electron bunch produced by the LWFA and compare them with that from a conventional linear accelerator.
\end{abstract}

Keywords: very high energy electrons (VHEEs), radiotherapy, depth-dose distribution, laser wakefield acceleration, laser-plasma accelerator, dose rate, treatment time, multi-beam arrangement

\section{INTRODUCTION}

Intensity-modulated radiotherapy (IMRT) using X-rays between 4 and $22 \mathrm{MeV}$ is currently the most flexible method of delivering a high radiation dose to a target volume while simultaneously protecting adjacent sensitive tissue. IMRT spreads the dose over a large volume of healthy tissue while concentrating maximum dose within the tumour volume. However, its efficacy is still questioned by some ${ }^{1}$ and it carries an increased risk of radiation induced malignancy. ${ }^{2,3}$ In contrast, advanced radiotherapy modalities such as proton and ion beam therapies have been shown to be effective because of the combination of their high penetration, favourable dose distributions within tissue, and increased radiobiological effectiveness. However, their cost is currently considered too high for wide availability because large infrastructures are required to transport beams to the patient. ${ }^{4}$ Recent investigations have shown that electrons with increased energy (up to $50 \mathrm{MeV}$ ) feature favourable dose distributions, particularly in combination with photon beams. ${ }^{5-7}$ Very high energy electron (VHEE) therapy using electron beams up to $250 \mathrm{MeV}$ was first considered by DesRosiers et al. ${ }^{8,9}$ as a viable and cost-effective alternative to intensity-modulated radiation therapy (IMRT) with photon and proton beams. Theoretical studies, based on the Monte Carlo (MC) methods, have demonstrated that VHEEs provide an implicit tool for scanning deep-seated lesions. The dosimetric characterisation of VHEE beams has already been performed in previous work. ${ }^{10-15}$ These studies conclude that main secondary products of the VHEE interactions with water molecules, neutrons and protons, should not significantly affect equivalent doses in comparison with photon treatments.

The treatment planning comparison studies provide necessary information for introduction of new radiotherapy modalities into clinical practice. Monte Carlo-aided simulations ${ }^{16-21}$ were carried out towards assessment of

\footnotetext{
Further author information: 1a) +44 141 5484305, karolina.kokurewicz@strath.ac.uk 1 b) +44 141 5485785, g.welsh@strath.ac.uk 1c) +44 141 5484305, enrico.brunetti@strath.ac.uk $1 \mathrm{~d}) \quad+44 \quad 141 \quad 5485785$, mark.wiggins@strath.ac.uk 1e) +44 $141 \quad 5483057, \quad$ dino@phys.strath.ac.uk 2a) +44 $141548 \quad 2263$, marie.boyd@strath.ac.uk 2b) +44 141 5482263, annette.sorensen@strath.ac.uk 3a) +44 $141 \quad 3306426$, anthony.chalmers@glasgow.ac.uk 4a) +44 20 89436589, $\quad$ giuseppe.schettino@npl.co.uk $\quad 4 b) \quad+44 \quad 20 \quad 89438548$, anna.subiel@npl.co.uk 5a) +1 317 9442524, cmdesros@iupui.edu
} 
treatment planning with VHEEs. The lower ratio of total dose to the target dose, higher dose rates (Gy/s) and therefore better conformity to the tumour volume and tissue sparing, comparable to traditional photon therapy with energies 6 and $16 \mathrm{MV}$, have been investigated and reported. Utilising the voxel-PENELOPE engine code as a preliminary treatment planning system (TPS) for very high energy electron therapy (VHEET) has been recently proposed. ${ }^{15,20}$ This is a significant move towards building a tool allowing further comprehensive studies for designing and planning of future VHEET systems. Yeboah and Sandison, following the current trends in radiotherapy quantified the performances of treatment plans using VHEEs versus photon and proton therapy. They have shown that the probability of complication-free tumour control for clinically relevant treatment plans decreases in the following order; intensity-modulated proton therapy (IMPT), VHEET and intensity-modulated X-ray therapy (IMXT), respectively. ${ }^{22}$ The feasibility of using intensity modulated VHEET and VHEE Pencil Beam Scanning (PBS) have been explored in more detail. ${ }^{15,23}$ The present research on VHEEs aims to investigate technical features, treatment quality, delivery and manipulation of VHEEs. ${ }^{24-27}$ To improve dose distribution and examine the radiobiological effectiveness (RBE) of VHEEs have yet to be explored.

\section{CURRENT RADIOTHERAPY MODALITIES VS. VHEES}

Figure 1 presents Monte Carlo calculated percentage depth dose (PDD) curves in $30 \times 30 \times 30 \mathrm{~cm}^{3}$ water phantom for various types of radiation with $10 \times 10 \mathrm{~cm}^{2}$ field size. Megavoltage photons (1a), due to the rapid increase to a peak dose in the shallow depths, produce low surface dose causing less damage to the skin. In IMRT the number of beam portals arranged in several angles around the patient, is used to create a dose plateau deep inside the tissue and a sharp dose contrast at the tumour edge (1b).

Clinical electron beams with energies in the range of 4-25 $\mathrm{MeV}$ (1c) have finite penetration range in tissue, after which the dose falls off rapidly. As such, this radiation modality is suitable for treatment of superficial lesions, and rarely in intraoperative radiotherapy to provide a boost to the tumour bed. Sub-cutaneous and visceral cancers are treated with electrons in this energy range in combination with megavoltage photon beams. ${ }^{28}$

Protons and heavy ions $(150-300 \mathrm{MeV})$ deposit most of their energy into a narrow region known as the Bragg peak (1d). Therefore, these beams have tremendous advantage over X-rays allowing precise localisation of dose in a tumour. The uniform dose region, in the longitudinal direction, referred to as Spread-out-Bragg Peak (SOBP) (1e), can be achieved by integrating the planar dose distributions created by altering the initial proton energy at the accelerator source. A high level of entrance dose results from the superposition of several narrow mono-energetic pencil-shaped beams during intensity modulated proton therapy (IMPT).

The depth of maximum dose deposited by $150 \mathrm{MeV}$ electron beams (1f) already exceeds the depth of most deep-seated tumours $(>10 \mathrm{~cm})$. For depths $\leq 5 \mathrm{~cm}$ VHEEs have been proven to exhibit penumbrae comparable to photons, ${ }^{9}$ and more uniform dose distribution at larger depths. VHEEs do not exhibit significant perturbations when propagating through tissues of varying densities, unlike photons ${ }^{9}$ and protons, ${ }^{29}$ which abruptly scatter and dissipate the dose within surrounding tissue. The dose distribution can be shaped by using non-overlapping multiple beams similar to those found in the IMRT scheme. For particle beams the probability of scattering events increases with depth. Electrons transfer most of their energy to tissue at the end of their range similar to protons. However the most intense scattering, at the Bragg peak, takes place beyond the patient since VHEEs exit the tissue before they terminate propagation.

\section{LASER PLASMA VS. CONVENTIONAL ACCELERATORS}

Over the past decade, significant efforts have been made to develop complex beam geometries and control the particle and radiation beams transport employing superconducting magnets. ${ }^{30-32}$ Additionally, this technology enables to produce more compact sources. However, the size of hadron therapy centres still remains large because of immense gantries required to transport the beam, long beamlines and extensive shielding. All of these translate into high construction and running costs. For instance, the price of building proton centre is 40 times higher than for LINAC X-ray facility. ${ }^{33}$ Therefore access to hadron therapy is limited what stimulates a search for more compact types of accelerators and alternative radiation modalities.

Very high energy electron therapy, proposed by DesRosiers et al. ${ }^{8}$ has a potential of becoming new radiation treatment method due to dosimetric advantages over conventional X-rays. However, the high cost and size of 


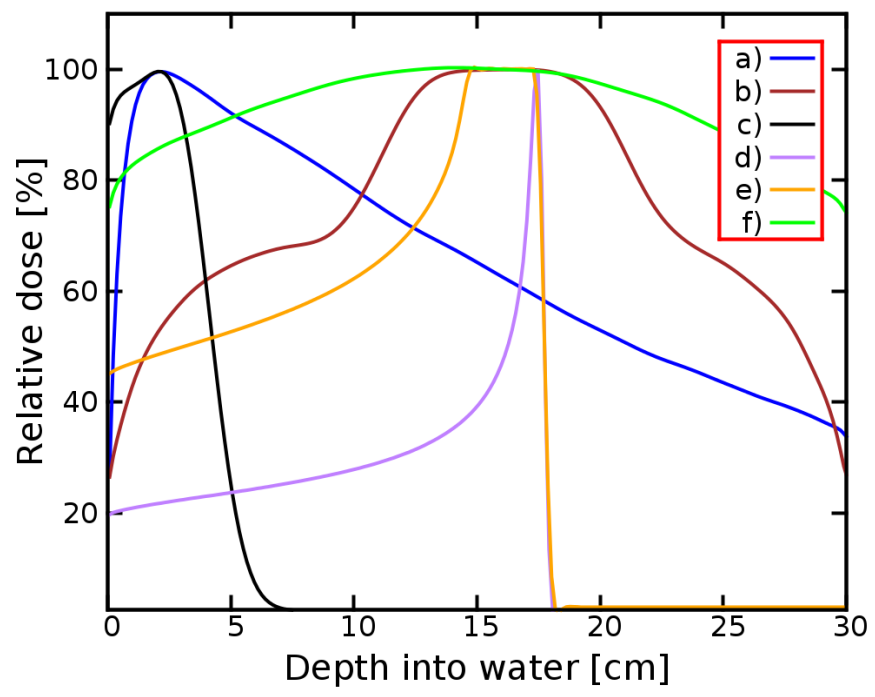

Figure 1. The percentage depth-dose distribution for different types of radiation: photons 6 MV (a), superposition of $25 \mathrm{MV}$ parallel opposed photon beams and perpendicular beam (b), $10 \mathrm{MeV}$ electrons (c), protons $160 \mathrm{MeV}$ (d) spread-out-Bragg-peak (e), $150 \mathrm{MeV}$ electrons (f).

LINAC-based VHEE accelerators has thwarted progress in developing the VHEE modality. In 1979 Tajima and Dawson ${ }^{34}$ introduced the concept of Laser Wakefield Acceleration (LWFA). This technology offers a much more compact accelerator footprint, requiring sufficient laser technology as the main driver. The development of table-top laser systems allows the true potential of LWFA to be explored. In 2004, three independent research groups $^{35-37}$ used the LWFA concept to demonstrate a new type of accelerator based on laser-driven plasma waves. In laser-plasma wakefield accelerators (LWFAs) background plasma electrons are trapped in the LWFA plasma bubble "structure" and get accelerated coherently to extremely high energies over several millimetres. High quality, mono-energetic, ultra-short duration, high peak current electron bunches are produced simply by placing a supersonic hydrogen or helium gas jet at the focus of an ultra-short pulse terawatt (TW) laser beam. ${ }^{38}$

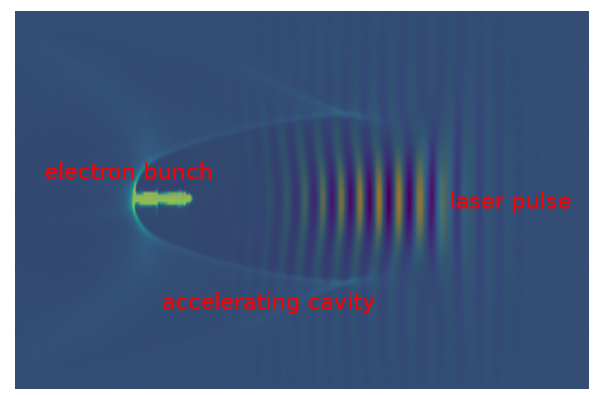

Figure 2. Electron bunch trapped in plasma cavity created behind the laser pulse during wakefield acceleration.

Typically $10^{18}-10^{19}$ electrons $/ \mathrm{cm}^{3}$ provide the space-charge fields of plasma of the order of $100 \mathrm{GV} / \mathrm{m}$, which is roughly 1000 times higher than in cavities of conventional accelerators. ${ }^{39}$ This allows to reduce the size of a 100-200 MeV accelerator from 10s of metres to just 1-2 mm exceeding currently existing energy limits. ${ }^{40,41}$ Conventional S-band LINACs typically produce electrons with few microsecond duration macro-pulses formed of $\approx 3 \mathrm{GHz}$ micro-pulse trains of $10-35$ ps duration and $\approx 30 \mathrm{pC}$ charge. In contrast, LWFA VHEE electrons have bunch durations of around 1 femtosecond $\left(10^{-15} \mathrm{~s}\right)$ at source, charges of $\approx 10 \mathrm{pC}$, and $\delta \mathrm{E} / \mathrm{E} \approx 1-10 \%$ energy spread. ${ }^{42}$ They currently operate at a repetition rate of up to $10 \mathrm{~Hz}$, but progress is being made to extend the rates to $1 \mathrm{kHz}$ (e.g. by using Coherent Amplifying Network (CAN) laser). ${ }^{43}$ 
Shortly after first experimental demonstration of the LWFA, these sources were considered as a potential way of producing VHEEs for radiotherapy. ${ }^{10}$ Simulations of $170 \mathrm{MeV}$ LWFA electrons were used to investigate an inverse treatment planning system for intensity-modulated treatments, which showed better target coverage than 6 MV IMXT. ${ }^{19}$

An example of an LWFA source used for radiobiology studies is the Advanced Laser-Plasma High-energy Accelerators towards X-ray (ALPHA-X) project at the University of Strathclyde ${ }^{14}$. The accelerator beamline is part of the various dedicated beamlines available across three shielded areas at the Scottish Centre for the Application of Plasma-based Accelerators (SCAPA). This state-of-the-art centre offers a suite of high peak-power laser systems to drive accelerators towards unique applications. SCAPA offers $40 \mathrm{TW}$ and $350 \mathrm{TW}$ peak-power laser systems across a range of accelerator type and technologies. SCAPA $40 \mathrm{TW}$ laser system delivers a laser pulse of $35 \mathrm{fs}$ pulse duration with a central wavelength of $800 \mathrm{~nm}$ at repetition rate up to $10 \mathrm{~Hz}$ and energy of $900 \mathrm{~mJ}$ on target and has already been used for dosimetry measurements of VHEEs. ${ }^{11,13}$ The ALPHA-X beam line is shown in Figure 3. The SCAPA $40 \mathrm{TW}$ laser beam coming directly from the compressor is focused at the entrance of a gas-jet by a spherical mirror (with focal length $80 \mathrm{~cm}$ ). The laser is focused to a focal spot diameter of $40 \mu \mathrm{m}\left(1 / \mathrm{e}^{2}\right.$ diameter) into a supersonic helium gas jet. The aberration-free focal spot can be achieved by proper alignment of the $\mathrm{f} / 18$ spherical mirror. When the laser beam interacts with plasma, electron beams with energy of at least $100 \mathrm{MeV}$ and $\delta \mathrm{E} / \mathrm{E} \approx 1 \%$ energy spread are produced. A setup of three miniature permanent magnet quadrupole (PMQ) lenses, are available preventing electron beam blow-up over long drift propagation as they pass through Lanex screens, used to monitor the transverse beam profile. The energy spectrum is measured by a spectrometer co-aligned with the beam line axis. Multiple Lanex screens imaged by high-resolution charge-coupled device (CCD) cameras are available to monitor the electron beam profile/pointing along the linear beamline.

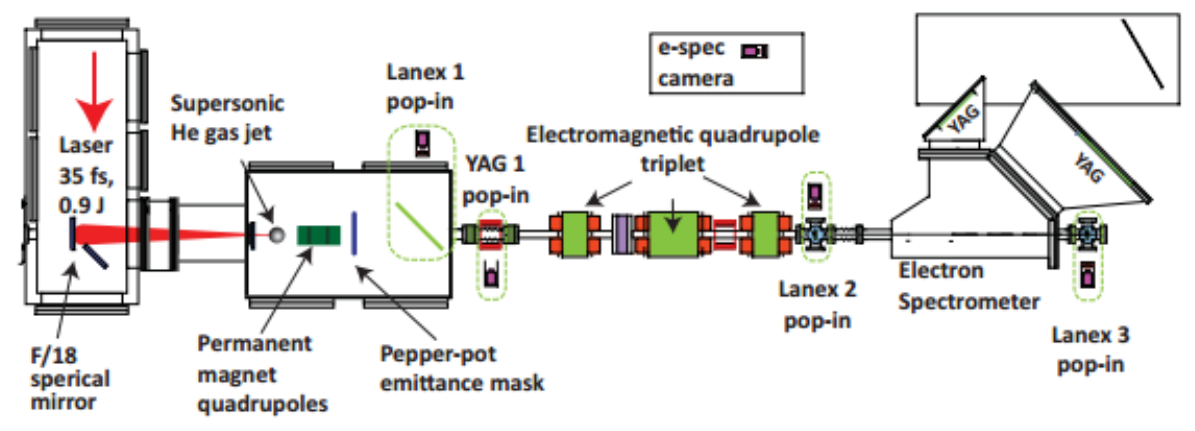

Figure 3. The ALPHA-X beamline.

The very short duration and high charge density of electron bunches produced by ALPHA-X (in the range of 5-10 pC) lead to very high energy beams ideally suited to VHEE studies.

\section{DOSE RATES AND TREATMENT TIME}

Several factors have a strong impact on tumour cell kill and tumour response outcome. Among these, dose rate and overall treatment time are pertinent and depend on accelerator capabilities. The dose-rate range in external radiotherapy extends from about $1.8 \mathrm{~Gy} / \mathrm{hr}$ to several $\mathrm{Gy} / \mathrm{min}$ and usually varies with the acceleration type, source to surface distance (SSD), energy and geometry. Commercial medical LINACs operate within dose rate up to $6 \mathrm{~Gy} / \mathrm{min}$ while cyclotrons for proton therapy deliver typically $2 \mathrm{~Gy}$ in less than 1 min. Patient comfort during the treatment and treatment accuracy are directly related with radiation treatment time. Treatment duration is established based on accelerator capabilities (dose rate regime), tolerance dose and recurrence time of normal cells. A number of studies have shown that early-stage cancer treated with a shorter and more intense radiation (e.g. accelerated hypofractionated whole breast irradiation) results in fewer side effects than the traditional six-week course. ${ }^{44-46}$ The ALPHA-X accelerator is capable to produce a bunch (shot) of electrons with a charge typically $10 \mathrm{pC}$ carrying $6.25 \times 10^{7}$ particles corresponding to $0.07 \mathrm{cGy}$. For a $10 \mathrm{~Hz}$ laser system the number of 
shots to deliver 2 Gy to the target (water) at $15 \mathrm{~cm}$ depth is around 3000 , this translates to approximately 5 minutes irradiation time and dose rates around $0.4 \mathrm{~Gy} / \mathrm{min}$. Therefore, efforts are being continued to increase the delivered charge of the produced electron bunches in order to increase dose rates. ${ }^{47}$ Proton therapy accelerators, on the other hand, with a built-in gantry systems are currently capable of delivering at a rate of roughly 3000 Bragg peaks per minute $(\approx 2 \mathrm{~Gy})$, equivalent to $50 \mathrm{~Hz},{ }^{48}$ this translates into delivery times per field of between a few seconds to 20-25 min. The arrangement of two opposite fields escalates the treatment time to 50 min.

\section{RADIOBIOLOGY}

Two mechanisms of cell kill are commonly identified path-ways in radiobiology: single and double DNA hit mechanisms. The former is associated to be responsible for sub-lethal DNA double-strand breaks (DSB) and thus chromosome aberration formation at the micrometre level, whereas the latter causes lethal damage by several chemical changes to DNA at the nanometre level. Normal tissue complication can result from the inactivation of stem cells by both mechanisms but tissue architecture or damage to vasculature could also play a role. In current clinical practice 1.8 - 3 Gy fractions are administrated for 5 days a week, up to the prescribed dose to the tumour area. The optimal combination of adequate killing of tumour clonogens and the time given to normal cells for convalescence, fractionation scheme, is decided by oncologist based on specification of the clinical case. It has been shown that irradiation with low dose rates in many fractions results in reduced cell killing due to fast proliferation of the tumour cells and enzymatic repair of sub-lesions in the tumour. ${ }^{49}$ The effects of dose-rates below the threshold at which micrometer-scale effects are still observable are studied based on the mechanism of DNA double strand breaks (DSBs), gene mutations, chromosomal aberrations and cell-cycle activation and apoptosis. The probability of killing the cell depend on the cell phase at the point when radiation is delivered. The cell is more radiosensitive in phases G2-phase and mitosis. In S phase, when DNA is being replicated, cells seem more able to repair DNA damage. However, the cell-cycle is a stochastic factor and depends upon the statistics of the number of cells found in the same phase.

In 1959 Bergonie and Tribondeau ${ }^{50}$ proved that increased sensitivity to radiation is seen in populations of cells that are highly proliferative with rapid cell divisions and less differentiated (i.e. stem cells are more sensitive than mature somatic cells). Tumour cells usually divide faster than normal cells thus there are more sensitive to radiation. Poorly-differentiated carcinomas are the most aggressive tumours or cancers in their final stage. Due to uncontrolled proliferation some areas within tumour are isolated from blood vessels and become hypoxic (more resistant). To achieve uncomplicated local regional control of cancer different types of cells/tissue need to be treated with various dose rates resulting in improved treatment efficiency. The dose rates which impact the biological response can be established based on the existing mathematical models; tumour control probability (TCP) and normal tissue complication (NTCP). The former is a probability of killing tumour cells after a certain total dose is delivered, whereas the later is defined as the probability that a certain percentage of the patient population will incure unfavourable side effects at a particular dose. Current LWFA technology, in contrast to conventional accelerators, are able to vary in repetition scaling (up to $10 \mathrm{~Hz}$ ) allowing alternating dose rate.

\section{FUTURE OUTLINE}

The LWFA accelerator is a promising and cost-effective alternative to produce VHEEs. Its compact size is dictated by the use of a non-ionising radiation source (laser light). Therefore, transport of the laser beam up to the accelerator head requires no additional shielding unlike in conventional accelerators. Furthermore, instead of traditional around 2-3 m long arrangement of RF cavities and bending magnets to accelerate and transport the beam, the gantry of a LWFA accelerator would contain laser optics for guiding the laser beam and the acceleration would take place in the plasma cell of less than $\mathrm{mm}^{41}$ length mounted in the accelerator head. The conceptual scheme of the future accelerator was proposed by Nakajima et al. ${ }^{51,52}$ Additional advantages of the LWFA design is the possibility to be developed into a multimodal machine. Laser-plasma electron bunches can be used to produce X-rays and protons through laser-solid target interactions (with the target placed in the accelerator head). However, production of protons, using laser-plasma technology, is currently very limited due to low efficiency of energy conversion (around 1\%) and, therefore, reduced beam quality.

The laser transport allows the optical geometry to be adapted to transport the laser to various fixed accelerators around the patient (multiple beams) and many treatment rooms (see Figure 4). A new dedicated 
beamline at SCAPA will distribute the LWFA produced radiation in a vertical orientation, as opposed to the LWFA community standard of a horizontal accelerator. Using this layout pioneering radiotherapy cell studies can be performed in a traditional patient style layout. In addition, SCAPA laser system and current LWFA technologies are able to vary in repetition scaling (up to $10 \mathrm{~Hz}$ ) allowing further investigation towards the future charge/dose rate modulation VHEET.

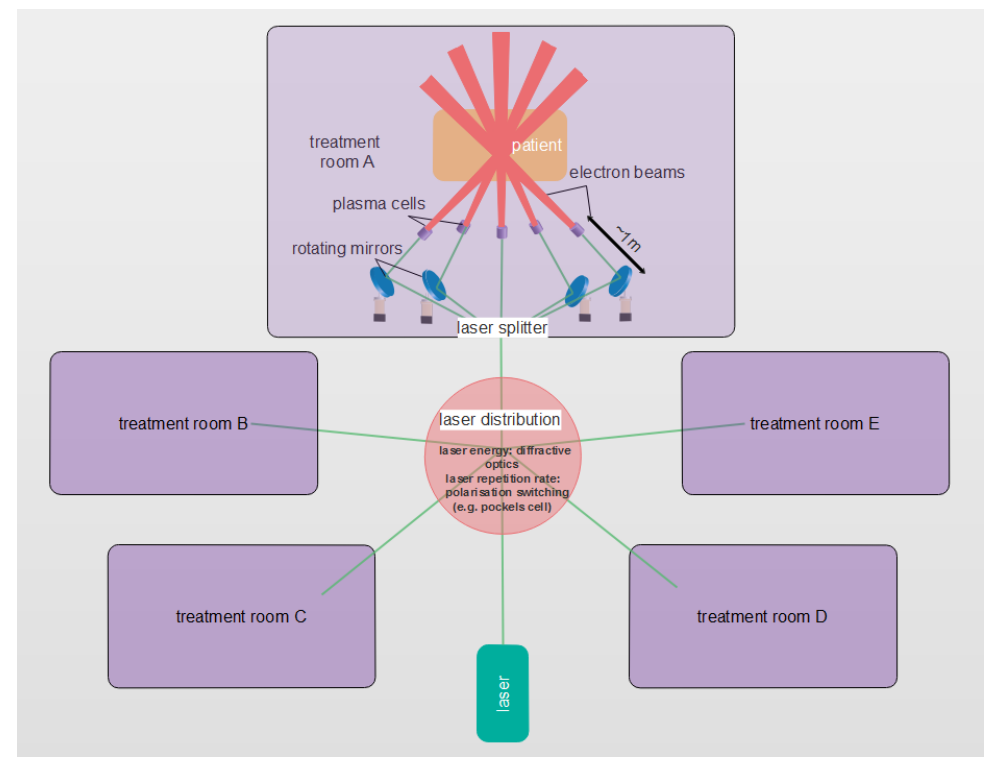

Figure 4. The conceptual scheme of medical centre for LWFA VHEET.

\section{CONCLUSIONS}

The ability to produce extremely short electron bunch durations from LWFA can provide a more effective way of killing cancer cells and reducing treatment time. The key challenges remain to identify the biological mechanisms following exposure to LWFA VHEE beams to understand their dose and dose-rate responsiveness and manifest clinically increased tumour control. The designed radiobiological models can be used to estimate the dose rates which impact the biological response. Based on the existing knowledge VHEEs administrated in the similar way to IMRT can provide similar tumour conformity and lower entrance dose in treatment of deep-seated tumours.

A combination of compacted, cost-effective technology and favourable dosimetric properties of VHEEs is a promising alternative which offers multiple options to design high precision, compact and cost-effective radiotherapy modality. Advanced optical technology allows to share one laser beam between multiple rooms in addition to providing an easy and stable guiding system with no shielding required. The electron beams can be delivered in a portable mode from robotic arm (similar to Cyber Knife) or gantry (similar to proton therapy but in more compact way). ${ }^{51,52}$ We propose a novel solution, the multiaccelerator arrangement in a single treatment room, which can allow many beams to be delivered at the same time. This mode could significantly reduce the radiation time and treatment uncertainties compared to X-ray therapy. The additional advantages of LWFA over conventional LINACs are the capability to produce different types of radiation (electrons, X-rays and protons) and control beam parameters from the source by changing parameters of the laser and the gas.

\section{ACKNOWLEDGEMENT}

This work has been supported by EPSRC EP/N028694/1, EP/J500094/1, STFC ST/H003754/1, CSO ETM/194, H2020 EC-GA LASERLAB-EUROPE 654148, EuCARD-2 312453 and EuPRAXIA 653782. The authors would like to thank the OSIRIS consortium (UCLA/IST) for the use of OSIRIS. Computational results were obtained using the EPSRC funded ARCHIE-WeSt High Performance Computer (www.archie-west.ac.uk) EPSRC grant no.EP/K000586/1. 


\section{REFERENCES}

[1] E. Glatstein, Intensity-modulated radiation therapy: the inverse, the converse, and the perverse, Seminars in Radiation Oncology 12(3):272-81, 2002.

[2] J. Ruben, S. Davis, C. Evans, P. Jones, F. Gagliardi, M. Haynes, A. Hunter, The effect of intensity-modulated radiotherapy on radiation-induced second malignancies, International Journal of Radiation Oncology, Biology, Physics 70(5):15306, 2008.

[3] S. Kry., M. Salehpour, D. Followill, M. Stovall, D. Kuban, R. White, I. Rosen, The calculated risk of fatal secondary malignancies from intensity-modulated radiation therapy, International Journal of Radiation Oncology, Biology, Physics 62(4):1195203, 2005.

[4] M. Goitein, M. Jermann, The relative costs of proton and X-ray radiation therapy, Clinical Oncology 15(1):37-50, 2003.

[5] M. Karlsson, B. Zackrisson, Exploration of new treatment modalities offered by high energy (up to $50 \mathrm{MeV}$ ) electrons and photons, Radiotherapy and Oncology 43(3):303-9, 1997.

[6] E. Korevaar, R. van Vliet, E. Woudstra, B. Heijmen, H. Huizenga, Sharpening the penumbra of high energy electron beams with low weight narrow photon beams, Radiotherapy and Oncology 48(2):213-20, 1998.

[7] E. Korevaar, B. Heijmen, E. Woudstra, H. Huizenga, A. Brahme, Mixing intensity modulated electron and photon beams: combining a steep dose fall-off at depth with sharp and depth-independent penumbras and flat beam profiles, Physics in Medicine and Biology 44(9):2171-81, 1999.

[8] C. DesRosiers, V. Moskvin, A. Bielajew, L. Papiez, 150-250 MeV electron beams in radiation therapy, Physics in Medicine and Biology 45(7):1781-805, 2000.

[9] C. M. DesRosiers, An evaluation of very high energy electron beams (up to $250 \mathrm{MeV}$ ) in radiation therapy, Doctoral dissertation, Purdue University, 2000.

[10] Y. Glinec, J. Faure, V. Malka, T. Fuchs, H. Szymanowski, U. Oelfke, Radiotherapy with laser-plasma accelerators: Monte Carlo simulation of dose deposited by an experimental quasimonoenergetic electron beam, Medical Physics 33(1):155-62, 2006.

[11] V. Moskvin, A. Subiel, C. Desrosiers, M. Wiggins, M. Maryanski, M. Mendonca, M. Boyd, A. Sorensen, S. Cipiccia, R. Issac, G. Welsh, E. Brunetti, C. Aniculaesei, D. A. Jaroszynski, Characterization of the very high energy electrons, 150-250 MeV (VHEE) beam generated by ALPHA-X laser wakefield accelerator beam line for utilization in Monte Carlo simulation for biomedical experiment planning, Medical Physics 39, 2012.

[12] O. Lundh, C. Rechatin, J. Faure, A. Ben-Ismal, J. Lim, C. De Wagter, W. De Neve, V. Malka, Comparison of measured with calculated dose distribution from a 120-MeV electron beam from a laser-plasma accelerator, Medical Physics 39(6):3501-8, 2012.

[13] A. Subiel, V. Moskvin, G. H. Welsh, S. Cipiccia, D. Reboredo, P. Evans, M. Partridge, C. DesRosiers, M. P. Anania, A. Cianchi, A. Mostacci, E. Chiadroni, D. Di Giovenale, F. Villa, R. Pompili, M. Ferrario, M. Belleveglia, G. Di Pirro, G. Gatti, C. Vaccarezza, B. Seitz, R. C. Isaac, F. Brunetti, S. M. Wiggins, B. Ersfeld, M. R. Islam, A. Sorensen, M. Boyd, D. A. Jaroszynski, Dosimetry of very high energy electrons (VHEE) for radiotherapy applications, Physics in Medicine and Biology 59(19):5811-29, 2014.

[14] A. Subiel, Feasibility studies on the application of relativistic electron beams from a laser plasma wakefield accelerator in radiotherapy. Thesis, University of Strathclyde, 2014.

[15] M. Bazalova-Carter, B. Qu, B. Palma, B. Hrdemark, E. Hynning, C. Jensen, P. G Maxim, B. W Loo, Treatment planning for radiotherapy with very high-energy electron beams and comparison of VHEE and VMAT plans, Medical Physics 42(5):2615-25, 2015.

[16] C. Yeboah, G. A. Sandison, V. Moskvin, Optimization of intensity-modulated very high energy (50-250 MeV) electron therapy, Physics in Medicine and Biology 47:1285-301, 2002.

[17] L. Papiez, C. DesRosiers, V. Moskvin, Very high energy electrons (50-250 MeV) and radiation therapy, Technology in Cancer Research and Treatment 1(2):105-10, 2002.

[18] C. DesRosiers, V. Moskvin, M. Cao, C. J. Joshi, M. Langer, Laser-plasma generated very high energy electrons in radiation therapy of the prostate, SPIE Proceedings 6881:688109-1, 2008.

[19] T. Fuchs, H. Szymanowski, U. Oelfke, Y. Glinec, C. Rechatin, J. Faure, V. Malka, Treatment planning for laser-accelerated very-high energy electrons, Physics in Medicine and Biology 54:3315-28, 2009. 
[20] V. Moskvin, F. Salvat, D. K. Stewart, C. M. DesRosiers, PENELOPE Monte Carlo engine for treatment planning in radiation therapy with very high energy electrons (VHEE) of 150-250 MeV, In IEEE Nuclear Science Symposium Conference Record 1961-6, 2010.

[21] M. Bazalova-Carter, M. Liu, B. A. Palma, M. Dunning, D. McCormick, E. Hemsing, J. Nelson, K. Jobe, E. Colby, A. C. Koong, S. Tantawi, V. Dolgashev, P. G. Maxim, B. Loo, Comparison of film measurements and Monte Carlo simulations of dose delivered with very high-energy electron beams in a polystyrene phantom, Medical Physics 42(4):1606-13, 2015.

[22] C. Yeboah, G. A. Sandison, Optimized treamtnet planning for prostate cancer comparing IMPT, VHEET and 15 MV IMXT, Physics in Medicine and Biology 47(13):2247-61, 2002.

[23] W. Loo, P. G. Maxim, V. A. Dolgashev, Pluridirectional very high electron energy radiation therapy systems and processes, U.S. Patent 13/765,017, 2013.

[24] B. Palma, M. Bazalova-Carter, B. Hrdemark, E. Hynning, B. Qu, B. W. Loo, P. G. Maxim, The effect of beam parameters on very high-energy electron radiotherapy: a planning study, Medical Physics 41(6):111, 2014.

[25] C. Jensen, B. Palma, B. Qu, P. G. Maxim, B. Hrdemark, E. Hynning, M. Bazalova-Carter, Radiation therapy with very high-energy electron (VHEE) beams in the presence of metal implants, Medical Physics 41(6):286-7, 2014.

[26] B. Palma, M. Bazalova-Carter, B. Hrdemark, E. Hynning, B. Qu, B. W. Loo, P. G. Maxim, Evaluation of the performance of very high-energy electron (VHEE) beams in radiotherapy: Five clinical cases, Medical Physics 42(6):3568, 2015.

[27] B. Palma, M. Bazalova-Carter, B. Hrdemark, E. Hynning, B. Qu, B. W. Loo, P. G. Maxim, Assessment of the quality of very high-energy electron radiotherapy planning, Radiotherapy and Oncology 119(1):154-8, 2016.

[28] M. Asell, S. Hydynmaa, S. Sderstrm, A. Brahme, Optimal electron and combined electron and photon therapy in the phase space of complication-free cure, Physics in Medicine and Biology 44(1):235-52, 1999.

[29] I. Das, V. Moskvin, L. Coutinho, C. Cheng, Dose Perturbation at Air-Tissue Interface in Proton Beam Therapy, Medical Physics 39(6):3824, 2012.

[30] Y. Iwata, K. Noda, T. Shirai , T. Murakami , T. Furukawa, Design of a superconducting rotating gantry for heavy-ion therapy, Physical Review Special Topics - Accelerators and Beams 15:044701, 2012.

[31] Y. Iwata, K. Noda, T. Shirai, T. Furukawa, and T. Murakami, Development of curved combined-function superconducting magnets for a heavy-ion rotating-gantry, IEEE Transactions on Applied Superconductivity 24(3):1051-8223, 2014.

[32] V. Derenchuk, "The ProNova SC360 gantry", modern hadron therapy gantry developments, Cockcroft Institute, Daresbury, U.K. , Tech. Rep., 2014

[33] A. B Keener, Arrests reveal debate about costs and benefits of proton therapy, Nature Medicine 20(10):1081, 2014.

[34] T. Tajima, J. M. Dawson, Laser electron accelerator, Physical Review Letters 43(4):267-70, 1979.

[35] S. Mangles, C. Murphy, Z. Najmudin, A. Thomas, J. Collier, A. Dangor, E. Divall, P. Foster, J. Gallacher, C. Hooker, D. Jaroszynski, A. Langley, W. Mori, P. Norreys, F. Tsung, R. Viskup, B. Walton, K. Krushelnick, Monoenergetic beams of relativistic electrons from intense laser-plasma interactions, Nature 431(7008):5358, 2004 .

[36] C. Geddes, Cs. Toth, J. van Tilborg, E. Esarey, C. Schroeder, D. Bruhwiler, C. Nieter, J. Cary, W. P. Leemans, High-quality electron beams from a laser wakefield accelerator using plasma-channel guiding, Nature 431(7008):538-41, 2004.

[37] J. Faure, Y. Glinec, A. Pukhov, S. Kiselev, S. Gordienko, E. Lefebvre, J.-P. Rousseau, F. Burgy, V. Malka, A laser-plasma accelerator producing monoenergetic electron beams, Nature 431(7008):541-4, 2004.

[38] D.A Jaroszynski, R Bingham, E Brunetti, B Ersfeld, J Gallacher, B van der Geer, R Issac, S.P Jamison, D Jones, M de Loos, A Lyachev, V Pavlov, A Reitsma, Y. Saveliev, G Vieux and S.M Wiggins, Radiation sources based on laser-plasma interactions, Philosophical Transactions of The Royal Society 364(1840):689$710,2006$. 
[39] M. Litos, E. Adli, W. An, C. I. Clarke, C. E. Clayton, S. Corde, J. P. Delahaye, R. J. England, A. S. Fisher, J. Frederico, S. Gessner, S. Z. Green, M. J. Hogan, C. Joshi, W. Lu, K. A. Marsh, W. B. Mori, P. Muggli, N. Vafaei-Najafabadi, D. Walz, G. White, Z. Wu, V. Yakimenko, G. Yocky, High-efficiency acceleration of an electron beam in a plasma wakefield accelerator, Nature 515(7525):92-5, 2014.

[40] X. Wang, R. Zgadzaj, N. Fazel, Z. Li, S. A. Yi, X. Zhang, W. Henderson, Y.-Y. Chang, R. Korzekwa, H.-E. Tsai, C.-H. Pai, H. Quevedo, G. Dyer, E. Gaul, M. Martinez, A. C. Bernstein, T. Borger, M. Spinks, M. Donovan, V. Khudik, G. Shvets, T. Ditmire, M. C. Downer, Quasi-monoenergetic laser-plasma acceleration of electrons to $2 \mathrm{GeV}$, Nature Communications 4:1988, 2013.

[41] W.P. Leemans, A.J. Gonsalves, H.-S. Mao, K. Nakamura, C. Benedetti, Multi-GeV electron beams from capillary-discharge-guided subpetawatt laser pulses in the self-trapping regime, Physical Review Letters 113(25):245002, 2014.

[42] S. M. Wiggins, R. P. Shanks, R. C. Issac, G. H. Welsh, M. P. Anania, E. Brunetti, G. Vieux, S. Cipiccia, B. Ersfeld, M. R. Islam, R. T. L. Burgess, G. Manahan, C. Aniculaesei, W. A. Gillespie, A. M. MacLeod, D. A. Jaroszynski, High guality electron beams from a laser wakefield accelerator, Plasma Physics and Controlled Fusion 52:124032, 2010.

[43] G. Mourou, B. Brocklesby, T. Tajima, J. Limpert, The future is fiber accelerators, Nature Photonics 7(4):258-61, 2013.

[44] T. J. Whelan, J. P.Pignol, M. N. Levine, J. A. Julian, R. MacKenzie, S. Parpia, W. Shelley, L. Grimard, J. Bowen, H. Lukka, F. Perera, A. Fyles, K. Schneider, S. Gulavita, C. Freeman, Long-term results of hypofractionated radiation therapy for breast cancer, The New England Journal of Medicine 362(6):51320, 2010.

[45] J. S. Haviland, J. R. Owen, J. A. Dewar, R. K. Agrawal, J. Barrett, P. J. Barrett-Lee, H. J. Dobbs, P. Hopwood, P. A. Lawton, B. J. Magee, J. Mills, S. Simmons, M. A. Sydenham, K. Venables, M. Bliss, J. R. Yarnold, START Trialists' Group, The UK Standardisation of breast radiotherapy (START) trials of radiotherapy hypofractionation for treatment of early breast cancer: 10-year follow-up results of two randomised controlled trials, The Lancet Oncology 14(11):1086-94, 2013.

[46] H. Versmessen, V. Vinh-Hung, H. Van Parijs, G. Miedema, M. Voordeckers, N. Adriaenssens, G. Storme, M. De Ridder, Health-related quality of life in survivors of stage I-II breast cancer: randomized trial of post-operative conventional radiotherapy and hypofractionated tomotherapy, BMC Cancer 12:495, 2012.

[47] M. Mirzaie, N. A. Hafz, S. Li, T. Sokollik, F. He, Y. Cheng, Z. Sheng, J. Zhang, Enhanced electron yield from a laser-plasma accelerator using high-Z gas jet targets, Review of Scientific Instruments 86(10):103502, 2015.

[48] AJ Lomax, T. Bhringer, A. Bolsi, D. Coray, F. Emert, G. Goitein, M. Jermann, S. Lin, E. Pedroni, H. Rutz, O. Stadelmann, B. Timmermann, J. Verwey, DC Weber, Treatment planning and verification of proton therapy using spot scanning: initial experiences, Medical Physics 31(11):3150-7, 2004.

[49] G.Hahn, M. Little, Plateau-phase cultures of mammalian cells: An in vivo model for human cancer, Current Topics in Radiation Research 49:285-92, 1968.

[50] J. Bergonie, L. Tribondeau, Interprtation de quelques rsultats de la radiothrapie et essai de fixation d' une technique rationnelle, Comptes-rendus de l'Acadmie des Sciences 143:983-4, 1906.

[51] K. Nakajima, J. Yuan, L. Chen, Z. Sheng, Laser-driven very high energy electron/photon beam radiation therapy in conjunction with a robotic system, Applied Sciences 5(1):1-20, 2015.

[52] K. Nakajima, Laser-dirven electron beam and radiation sources for basic, medical and industrial sciences, Proceedings of the Japan Academy 91(6):223-45, 2015. 